\title{
Spontaneous CPT Violation in Confined QED
}

\author{
E. J. Ferrer ${ }^{1}$, V. de la Incera ${ }^{1}$, and A. Romeo ${ }^{2}$ \\ ${ }^{1}$ Department of Physics, State University of New York at Fredonia, \\ Houghton Hall 118, Fredonia, NY 14063, USA \\ ${ }^{2}$ Institute for Space Studies of Catalonia, CSIC, Edif. Nexus, Gran \\ Capita 2-4, 08034 Barcelona, Spain.
}

\begin{abstract}
Symmetry breaking induced by untwisted fermions in QED in a nonsimply connected spacetime with topology $S^{1} \times R^{3}$ is investigated. It is found that the discrete CPT symmetry of the theory is spontaneously broken by the appearance of a constant vacuum expectation value of the electromagnetic potential along the direction of space periodicity. The constant potential is shown to be gauge nonequivalent to zero in the nonsimply connected spacetime under consideration. Due to the symmetry breaking, one of the electromagnetic modes of propagation is massive with a mass that depends on the inverse of the compactification length. As a result, the system exhibits a sort of topological directional superconductivity.
\end{abstract}

Symmetry breaking in non-trivial spacetime, where the curvature, as well as the topology, can play an important role, has received much attention in the last years because of its possible relevance for cosmology in the early universe.

It is well known that the global properties of the spacetime, even if it is locally flat, can give rise to new physics. The seminal discovery in this direction is the so called Casimir effect [1], that is, the existence of an attractive force between neutral parallel perfectly conducting plates. In this phenomenon the attractive force appearing between the plates is mediated by the zero-point fluctuations of the electromagnetic field in vacuum. Thus, the Casimir force is interpreted as a macroscopic manifestation of the vacuum structure of the quantized fields in the presence of domains restricted by boundaries or nontrivial topologies [2].

A characteristic of quantum field theory in spacetime with non-trivial topology is the possible existence of nonequivalent types of fields with the same spin [3]. In particular, for a fermion system in a space-time which is locally flat but with topology represented by the domain $S^{1} \times R^{3}$ (i.e. a Minkowskian space with one of the spatial dimensions compactified in a circle $S^{1}$ of finite length $a$ ), the non-trivial topology is transferred into periodic boundary conditions for untwisted fermions or antiperiodic boundary conditions for twisted fermions

$$
\psi(t, x, y, z-a / 2)= \pm \psi(t, x, y, z+a / 2)
$$

In (1) the compactified dimension with length $a$ has been taken along the $\mathcal{O Z}$-direction.

Quantum electrodynamics (QED) with photons coupled to untwisted fermions or to a combination of twisted and untwisted fermions is an unstable theory [- [1], [5]. The instability arises due to polarization effects of untwisted electrons that change the propagation of electromagnetic waves giving rise to tachyonic electromagnetic modes [4], [5]. In Ref. [5] it was speculated that such instability might indicate that a stable vacuum configuration with nonzero expectation value of the strength tensor $F_{\mu \nu}$ could appear.

For self-interacting scalar fields the space periodicity can also produce instabilities causing a massless field to become massive [6], [7]. The acquired mass depends on the periodicity length, so the phenomenon is called topological mass generation.

Our main goal in this paper is to investigate the symmetry breaking induced by the non-trivial topology $S^{1} \times R^{3}$ in the case of QED with untwisted fermions. We will show that the stable vacuum configuration is given by a constant electromagnetic potential $A_{3}$ along the compactified dimension. As discussed below, even though such a vacuum configuration has $F_{\mu \nu}=0$, it cannot be gauged to zero, because the gauge transformation that would be needed does not respect the periodicity of the function space in the $S^{1} \times R^{3}$ domain. This is a sort of Aharonov-Bohm effect which takes place due to the non-simply connected topological structure of the considered spacetime. The nonzero vev of the electromagnetic potential spontaneously breaks the discrete CPT symmetry of the theory. Accordingly, the $A_{3}$ field acquires a topological mass and no tachyonic mode remains. The results here reported can be of interest for theories with extra dimensions on which some of them are compactified, and for condensed matter quasi-planar systems.

The existence of constant gauge potentials with physical meaning are known in statistics, where $A_{0}$ cannot be gauged away by reasons similar to those pointed out above [8]. The physical relevance of constant condensates of zero-components of gauge fields has been investigated in the context of many-particle electroweak theory in Refs. [9], [10].

Let us consider the QED action in a spacetime domain with compactified dimension of length $a$ in the $\mathcal{O Z}$-direction 


$$
\mathcal{S}=\int_{-a / 2}^{a / 2} d x_{3} \int_{-\infty}^{\infty} d x_{0} d^{2} x_{\perp}\left[-\frac{1}{4} F_{\mu \nu}^{2}+\bar{\psi}(i \not \partial-e \not A-m) \psi\right]
$$

The action (2), together with the boundary conditions (11), represents a system of photons and electrons confined between two infinite parallel planes perpendicular to the $\mathcal{O} \mathcal{Z}$-direction.

When this confined QED action is considered for untwisted fermions, the effect of vacuum polarization upon photon propagation yields a tachyonic mass for the third component of the photon field [4]. Tachyonic modes in a quantum theory are an indication that the vacuum under consideration is not really the physical one. A tachyonic mode is therefore related to some symmetry breaking mechanism which leads to the true physical vacuum. It follows that some symmetry breaking should then occur in the unstable theory (2) of photons interacting with untwisted fermions.

Henceforth we restrict our analysis to this unstable case. To find the physical vacuum that stabilizes the untwisted fermion theory, we propose the following ansatzt]

$$
\bar{A}_{\nu}=\Lambda \delta_{\nu 3}
$$

for the vacuum solution, with $\Lambda$ an arbitrary constant that will be determined from the minimum equation of the effective potential. Notice that a constant electromagnetic potential in the direction of the periodicity $(\nu=3$ in our case), although seemingly physically equivalent to the pure vacuum $\bar{A}_{\nu}=0$, since both have $F_{\mu \nu}=0$, cannot be gauged to zero. The reason is that due to the periodicity of the fields in the $S^{1} \times R^{3}$ space, the gauge transformations $A_{\mu} \rightarrow A_{\mu}-\frac{1}{e} \partial_{\mu} \alpha$ are restricted to those satisfying $\alpha\left(x_{3}+a\right)=\alpha\left(x_{3}\right)+2 l \pi, l \in Z$ [8]. However, the gauge transformation $\alpha(x)=(x \cdot n) e \Lambda$ connecting the constant field configuration (3) with zero does not satisfy the required periodicity condition unless $\Lambda$ satisfies

$$
\Lambda=\frac{2 l \pi}{e a}, \quad l \in Z
$$

Let us consider then the one-loop effective potential of the theory (2) around the vacuum configuration (3)

$$
V=-\frac{1}{2} a^{-1} \ln \left(\operatorname{Det} \bar{G}^{-1}\right)
$$

Here $\operatorname{Det} \bar{G}^{-1}=\mathbb{Z}^{4} p \operatorname{det} \bar{G}^{-1}=\sum_{p_{3}} \int d^{3} p \operatorname{det} \bar{G}^{-1}$, with $p_{3}=2 n \pi / a,(n=0, \pm 1, \pm 2, \ldots)$ being the discrete frequencies associated with periodic fermions. The fermion inverse Green's function in the background $\Lambda$ is

$$
\bar{G}^{-1}=\gamma \cdot \bar{p}+m
$$

with $\bar{p}_{\mu}=\left(p_{0}, \mathbf{p}_{\perp}, p_{3}-e \Lambda\right)$. After summing in $p_{3}$ we obtain

$$
V(\Lambda)=-\int_{-\infty}^{\infty} \frac{d^{3} \widehat{p}}{(2 \pi)^{3}}\left[\varepsilon_{p}+2 a^{-1} \operatorname{Re} \ln \left(1-e^{-a\left(\varepsilon_{p}-i e \Lambda\right)}\right)\right] .
$$

The extremum of the effective potential (7) satisfies

$$
\left.\frac{\partial V(\Lambda)}{\partial \Lambda}\right|_{\Lambda=\Lambda_{\min }}=-\int_{-\infty}^{\infty} \frac{d^{3} \widehat{p}}{(2 \pi)^{3}} \frac{2 e^{-a \varepsilon_{p}} \sin (a e \Lambda)}{1+e^{-2 a \varepsilon_{p}}-2 e^{-a \varepsilon_{p}} \cos (a e \Lambda)}=0
$$

The solution to (8) is $\Lambda=\frac{l \pi}{e a}, l \in Z$. Nevertheless, the minimum condition $\partial^{2} V\left(\Lambda_{\min }\right) / \partial^{2} \Lambda>0$ is only satisfied by the subset

$$
\Lambda_{\min }=\frac{(2 l+1) \pi}{e a}, \quad l \in Z
$$

\footnotetext{
${ }^{1}$ This is a natural ansatz given that the $\mathcal{O} \mathcal{Z}$-direction is the only distinguished direction in the $S^{1} \times R^{3}$ space under consideration, and that the $A_{\mu}$ field obeys periodic boundary conditions along the $\mathcal{O Z}$-direction.
} 
The elements in the set of minimum solutions (9), are gauge equivalent since they are all connected by allowed gauge transformations $\left(\alpha\left(x_{3}+a\right)=\alpha\left(x_{3}\right)+2 l \pi\right)$. It should be pointed out, however, that the solutions (9) are gauge nonequivalent to the trivial vacuum $\Lambda=0$, since none of them satisfies (4).

Substituting with the minimum solution (9) in Eq. (17) we obtain in the $a m \ll 1$ approximation that the effective potential of untwisted fermions reduces to

$$
V\left(\Lambda_{\min }\right)=-\frac{7 \pi^{2}}{360 a^{4}}
$$

which coincides with the result reported for twisted fermions in Ref. [4]. Thus, the vacuum energy of both classes of fermions coincides if the corresponding correct vacuum solution is used.

As discussed above, the appearance of the constant vacuum solution $\bar{A}_{\nu}=\Lambda_{\min } \delta_{\nu 3}$ at the one-loop level must be associated with symmetry breaking. It is easy to corroborate that if the gauge field in the action (2) is shifted by $A_{\mu}(x) \rightarrow A_{\mu}(x)-\Lambda_{\min } \delta_{\mu 3}$, a new term $e \Lambda_{\min } \psi \gamma_{3} \bar{\psi}$ emerges violating the CPT symmetry

$$
x_{\mu}=-x_{\mu}, \quad A_{\mu}(x)=-A_{\mu}(-x), \quad \psi(x)=-i \psi(-x) \gamma_{5} \gamma_{0}
$$

of the original action. We stress that the vacuum solution (9) breaks the CPT invariance by breaking each discrete symmetry separately, while maintaining CP or any other product of two symmetries intact.

We conclude that the spatial compactification leads to spontaneous CPT symmetry breaking with vacuum configuration given by a constant electromagnetic potential whose amplitude increases with the decreasing of the compactification radius as $1 / a$.

Let us find the electromagnetic modes' masses in the background of the new vacuum (9). With this end, the general structure of the electromagnetic field Green's function in the $S^{1} \times R^{3}$ space should be considered

$$
\Delta_{\mu \nu}(k)=P\left(g_{\mu \nu}-\frac{k_{\mu} k_{\nu}}{k^{2}}\right)+Q\left[\frac{k_{\mu} k_{\nu}}{k^{2}}-\frac{k_{\mu} n_{\nu}+n_{\mu} k_{\nu}}{(k \cdot n)}+\frac{k^{2} n_{\mu} n_{\nu}}{(k \cdot n)^{2}}\right]+\frac{\alpha}{k^{4}} k_{\mu} k_{\nu},
$$

Here, due to the breaking of Lorentz invariance, in addition to the usual tensor structures $k_{\mu}$ and $g_{\mu \nu}$, a spacelike unit vector $n^{\mu}=(0,0,0,1)$, pointing in the direction of the periodicity, must be introduced. In Eq. (12) $\alpha$ is a gauge fixing parameter corresponding to the gauge condition $\frac{1}{\alpha} \partial_{\mu} A_{\mu}=0$, and the coefficients $P$ and $Q$ are given by

$$
P=\frac{1}{k^{2}+\Pi_{0}}, \quad Q=-\frac{\Pi_{1}}{\left(k^{2}+\Pi_{0}\right)\left\{k^{2}+\Pi_{0}-\Pi_{1}\left[k^{2} /(k \cdot n)^{2}+1\right]\right\}},
$$

The parameters $\Pi_{0}, \Pi_{1}$, are the coefficients of the polarization operator $\Pi_{\mu \nu}$, whose general structure is

$$
\Pi_{\mu \nu}(k)=\Pi_{0}\left(g_{\mu \nu}-\frac{k_{\mu} k_{\nu}}{k^{2}}\right)+\Pi_{1}\left[\frac{k_{\mu} k_{\nu}}{k^{2}}-\frac{k_{\mu} n_{\nu}+n_{\mu} k_{\nu}}{(k \cdot n)}+\frac{k^{2} n_{\mu} n_{\nu}}{(k \cdot n)^{2}}\right]
$$

The coefficient $\Pi_{1}$ is absent in the usual flat space case with trivial topology. It arises here because of the explicit breaking of the Lorentz invariance in the $S^{1} \times R^{3}$ spacetime. The situation is similar to that in statistical quantum field theory [11], where the presence of a medium breaks the Lorentz invariance giving rise to the compactification of the time variable. The role of $n_{\mu}$ in the finite temperature case is played by the four-velocity of the medium $u_{\mu}$.

From (14) it is easy to see that the polarization operator coefficients can be expressed in terms of the two independent tensor components $\Pi_{33}$ and $\Pi_{00}$ through the relations

$$
\begin{aligned}
& \Pi_{0}=\frac{k^{2}}{\kappa}\left[k_{3}^{2} k_{0}^{2} \Pi_{33}-\widehat{k}^{4} \Pi_{00}\right] \\
& \Pi_{1}=\frac{k^{2} k_{3}^{2}}{\kappa}\left[\mathbf{k}^{2} \Pi_{33}-\widehat{k}^{2} \Pi_{00}\right]
\end{aligned}
$$

where we are using the notation $\widehat{k}_{\mu} \equiv\left(k_{0}, \mathbf{k}_{\perp}, 0\right)$ and $\kappa=\widehat{k}^{2}\left(\mathbf{k}^{2} \widehat{k}^{2}-k_{0}^{2} k_{3}^{2}\right)$.

The electromagnetic modes' masses are found from the poles of the Green's function (12). From (13), (15) and (16) we have that for photons propagating in a direction perpendicular to the direction of periodicity $\left(k_{3}=0\right)$ the dispersion relations are

$$
k_{0}^{2}-\mathbf{k}_{\perp}^{2}-\frac{\widehat{k}^{2}}{\mathbf{k}_{\perp}^{2}} \Pi_{00}=0
$$




$$
k_{0}^{2}-\mathbf{k}_{\perp}^{2}-\Pi_{33}=0
$$

From Eqs. (17)-(18) we see that the values of the $\Pi_{00}$ and $\Pi_{33}$ polarization operator components in the limit $k_{3}=0$, $|\widehat{k}| \rightarrow 0$ are crucial for the properties of the electromagnetic field propagation in this non-trivial space, since they play the role of the square masses of the electromagnetic modes $\left(M_{1}^{2}=\left(\widehat{k}^{2} / \mathbf{k}_{\perp}^{2}\right) \Pi_{00}, M_{2}^{2}=\Pi_{33}\right)$. The appearance of the electromagnetic tachyonic mass for untwisted electrons comes precisely from the fact that in the $a|\widehat{k}| \ll a m \ll 1$ limit, those polarization operator components, calculated on the trivial electromagnetic vacuum $(\Lambda=0)$, are given by

$$
\begin{gathered}
\Pi_{33}\left(k_{3}=0, \widehat{k}\right) \simeq-\frac{8 e^{2}}{a^{2}}\left[\frac{1}{12}-\frac{\xi}{4}+\mathcal{O}\left(\xi^{2}\right)\right]+\mathcal{O}\left(\widehat{k}^{2}\right) \\
\Pi_{00}\left(k_{3}=0, \widehat{k}\right) \simeq \frac{e^{2}}{3 \pi^{2}} \mathbf{k}_{\perp}^{2}\left[\frac{1}{4 \xi}+\frac{1}{2} \ln \xi+\mathcal{O}\left(\xi^{0}\right)\right]+\mathcal{O}\left(k_{\perp}^{4}\right)
\end{gathered}
$$

where $\xi=a m / 2 \pi \ll 1$. The leading term in Eq. (19) coincides with the one reported in Ref. 雊, after a factor correction 2 previously pointed out in Ref. 12]. From (19) and (20) we have that in the $k_{3}=0,|\widehat{k}| \rightarrow 0$ limit the electromagnetic modes' masses in the trivial vacuum are $M_{1}^{2}=0$ and $M_{2}^{2} \leq 0$ (tachyonic mass) respectively.

To investigate the modification produced in the dispersion equations (17)-(18) by the nontrivial vacuum (9) the polarization operator components $\Pi_{00}, \Pi_{33}$ must be calculated in the background of the non-trivial vacuum state. With this aim, we start from the free-fermion propagator for untwisted fermions in the non-trivial vacuum

$$
G\left(x-x^{\prime}\right)=\frac{1}{(2 \pi)^{3} a} \Varangle d^{4} p \exp \left[i p \cdot\left(x-x^{\prime}\right)\right] G(\widetilde{p})
$$

with

$$
G(\widetilde{p})=\frac{\not{p}-m}{\widetilde{p}^{2}-m^{2}+i \epsilon}, \quad \widetilde{p}_{\mu}=\left(p_{0}, \mathbf{p}_{\perp}, p_{3}-e \Lambda_{\min }\right)
$$

The polarization operator components corresponding to the Green's functions (21) can be found to order- $e^{2}$ in the coupling constant from

$$
\Pi_{\mu \nu}^{\Lambda}(k)=-\frac{4 i e^{2}}{(2 \pi)^{3} a} \sum d^{4} p \frac{\widetilde{p}_{\mu}\left(\widetilde{p}_{\nu}-k_{\nu}\right)+\widetilde{p}_{\nu}\left(\widetilde{p}_{\mu}-k_{\mu}\right)-\widetilde{p} \cdot(\widetilde{p}-k) g_{\mu \nu}+m^{2} g_{\mu \nu}}{\left(\widetilde{p}^{2}-m^{2}\right)\left[(\widetilde{p}-k)^{2}-m^{2}\right]}
$$

After summation in $p_{3}$ we find for the polarization operator components entering in the dispersion relations (17)-(18)

$$
\begin{gathered}
\Pi_{33}^{\Lambda}\left(k_{3}=0, \widehat{k}\right)=-\frac{e^{2}}{\pi^{3}} \int d \widehat{p} \frac{2 \varepsilon_{p}^{2}-\widehat{p} \cdot \widehat{k}}{\varepsilon_{p}\left(\varepsilon_{p-k}^{2}-\varepsilon_{p}^{2}\right)} n(p) \\
\Pi_{00}^{\Lambda}\left(k_{3}=0, \widehat{k}\right)=\frac{e^{2}}{\pi^{3}} \int d \widehat{p} \frac{2 p_{4}\left(p_{4}-k_{4}\right)+\widehat{p} \cdot \widehat{k}}{\varepsilon_{p}\left(\varepsilon_{p-k}^{2}-\varepsilon_{p}^{2}\right)} n(p)
\end{gathered}
$$

where $\varepsilon_{p}=\sqrt{\widehat{p}^{2}+m^{2}}, \varepsilon_{p-k}=\sqrt{(\widehat{p}-\widehat{k})^{2}+m^{2}}$, and $n(p)=\left[1-\exp a\left(\varepsilon_{p} \pm i e \Lambda_{\min }\right)\right]^{-1}=\left(1+\exp a \varepsilon_{p}\right)^{-1}$. Finally, evaluating the integrals in (24), 25) and considering the $a|\widehat{k}| \ll a m \ll 1$ limit, we obtain

$$
\Pi_{33}^{\Lambda}\left(k_{3}=0, \widehat{k}\right) \simeq \frac{e^{2}}{a^{2}}\left[\frac{1}{3}+\mathcal{O}\left(\xi^{2}\right)\right]+\mathcal{O}\left(\widehat{k}^{2}\right)
$$

\footnotetext{
${ }^{2}$ The result in Eq. (4.9) of Ref. [⿴囗 sh should be multiplied by 2 .
} 


$$
\Pi_{00}^{\Lambda}\left(k_{3}=0, \widehat{k}\right) \simeq \frac{e^{2}}{3 \pi^{2}} k_{\perp}^{2}\left[\frac{1}{2} \ln \xi+\mathcal{O}\left(\xi^{0}\right)\right]+\mathcal{O}\left(k_{\perp}^{4}\right)
$$

It can be seen that (26) coincides with the result reported for twisted fermions in trivial vacuum in Ref. 何. Using the results (26) and (27) in the dispersion equations (17)-(18) we find that the electromagnetic modes propagate on the nontrivial vacuum with new masses $M_{1}^{2}[\Lambda]=0$ and $M_{2}^{2}[\Lambda]=e^{2} / 3 a^{2}>0$. It is interesting to notice that if we make the change $a \rightarrow \beta$ ( $\beta$ the inverse temperature) in $M_{2}^{2}[\Lambda]$, then we would formally obtain the well known result in statistical QED [11] of a Debye screening mass.

Then, we conclude that in the new vacuum state no tachyon is present. Instead, a massive electromagnetic mode, with a mass that depends on the inverse of the compactification radius $a$, arises. When the compactification length is taken to infinity $(a \rightarrow \infty)$, the flat-space QED theory is regained with zero photon mass. Moreover, the polarization of photons in the presence of twisted or untwisted fermions will be equivalent at least up to the one-loop level. However, they take place in different vacuums, the trivial one for twisted fermions and the nontrivial $\bar{A}_{3}=\frac{\pi}{e a}$ for untwisted ones.

The existence of a massive electromagnetic mode in confined QED for both untwisted and twisted fermions has also implications for the magnetic response of the system. To understand this, let us consider the modified Maxwell equation in linear response theory

$$
\left[\square \delta_{\mu \nu}-\lambda_{M}^{-2} \delta_{3 \mu} \delta_{3 \nu}\right] A_{\nu}=e J_{\mu}
$$

taken in the Lorentz gauge $\partial_{\mu} A^{\mu}=0$. In Eq. (28), we introduced the magnetic length $\lambda_{M}=1 / M_{2}[\Lambda]$. Considering an external static and constant current $I$ flowing inside the confined space along the $\mathcal{O} \mathcal{Z}$-axis, the current density in Eq. (28) becomes $e J_{3}(x)=I \delta^{2}\left(\mathbf{x}_{\perp}\right)$. Then, the induced potential, which is a solution of (28) with periodic boundary conditions in the $\mathcal{O Z}$-direction, will be

$$
A_{3}(x)=-\frac{I}{2 \pi} K_{0}\left[\left|\mathbf{x}_{\perp}\right| / \lambda_{M}\right]
$$

where $K_{0}$ denotes a modified Bessel function of the third kind. In the $\left|\mathbf{x}_{\perp}\right| \gg \lambda_{M}$ limit we obtain for the corresponding magnetic field

$$
\mathbf{B}(r)=\frac{I}{2 \sqrt{2 \pi}} \frac{\exp \left(-r / \lambda_{M}\right)}{\sqrt{r \lambda_{M}}} \widehat{\theta}
$$

with $r=\left|\mathbf{x}_{\perp}\right|$ and $\widehat{\theta}$ denoting the azimuth-angle unit vector in cylindrical coordinates. From (30) we see that an azimuthal magnetic field will be screened along the radial direction, in a distance equal to the inverse of the topological mass $M_{2}[\Lambda]$. When the separation between the two infinite parallel plates decreases, the screening effect increases.

Our results indicate that confined QED exhibits a sort of topological directional superconducting behavior, with a Meissner effect taking place for magnetic fields induced by electric currents flowing in the direction of the compactified dimension. This result could find applications in high- $\mathrm{T}_{C}$ superconductivity where, as it is well known, the system is confined to a quasi-two-dimensional space.

\section{Acknowledgments}

It is a pleasure for two of the authors (EJF and VI) to express their gratitude to the Institute for Spatial Studies of Catalonia and to the University of Barcelona for the warm hospitality extended to them during the time this work was completed. This work has been supported in part by NSF grant PHY-9722059 (EJF and VI), NSF POWRE grant PHY-9973708 (VI).

[1] H. B. G. Casimir, Proc. K. Ned. Akad. Wet. 51, (1948) 793.

[2] For a review see: K. A. Milton , 1999; The Casimir Effect: Physical Manifestations of Zero-Point Energy, Invited Lecture at the 17th Symposium of Theoretical Physics, Seoul National University, Korea, June 29-July 1, 1998 (hep-th/9901011).

[3] C. J. Isham, Proc. R. Soc. A 362 (1978) 383; S. J. Avis and C. J. Isham, Proc. R. Soc. A 363 (1978) 581; C. J. Isham, Proc. R. Soc. A 364 (1978) 591; R. Banach, J. Phys. A: Math. Gen. 14 (1981) 901.

[4] L. H. Ford, Phys. Rev. D 21 (1980) 933.

[5] L. H. Ford, Phys. Rev. D 22 (1980) 3003. 
[6] L. H. Ford and T. Yoshimura, Phys. Lett. A 368 (1979) 305.

[7] D. J. Toms, Phys. Rev. D 21 (1980) 928.

[8] N. Batakis and G. Lazarides, Phys. Rev. D 18 (1978) 4710; A. M. Polyakov, Phys. Lett. B 72 (1978) 477; I. Affleck, Nucl. Phys. B 162 (1980) 461; D. Gross, R. Pisarski and L. Yaffe, Rev. Mod. Phys. 53 (1981) 43; N. Weiss, Phys. Rev. D 24 (1981) 475; D 25 (1982) 2667; A. Actor, Phys. Rev. D 27 (1983) 2548; Ann. Phys. 159 (1985) 445; J. Phys. A33 (2000) 4585 .

[9] A. D. Linde, Phys. Lett. B 86 (1979) 39.

[10] E. J. Ferrer, V. de la Incera and A. E. Shabad, Phys. Lett. B 185 (1987) 407; Nucl. Phys. B 309 (1988) 120.

[11] E. S. Fradkin, Proceedings of Quantum Field Theory and Hydrodynamics, P. N. Lebedev Physical Institute, Vol. 29, 7 (1965, Moscow Nauka) (Engl. Transl., 1967 New York: Consultant Bureau).

[12] D. J. Toms, Phys. Lett. B 126 (1983) 445. 